\title{
Indications for percutaneous endoscopic gastrostomy and survival in old adults
}

\author{
Anna Malmgren', Gunnel Wärn Hede ${ }^{2}$, Brita Karlström³, \\ Tommy Cederholm ${ }^{4}$, Per Lundquist ${ }^{1,5}$, Mikael Wirén ${ }^{1,5}$ and \\ Gerd Faxén-Irving ${ }^{6} *$
}

\begin{abstract}
'Department of Surgical and Medical Gastroenterology and Hepatology, Karolinska University Hospital, Stockholm, Sweden; 'Department of Neurobiology, Care Sciences and Society, Division of Nursing, Karolinska Institutet, Karolinska University Hospital, Stockholm, Sweden; ${ }^{3}$ Department of Food, Nutrition and Dietetics, Uppsala University, Uppsala, Sweden; ${ }^{4}$ Department of Public Health and Caring Sciences, Clinical Nutrition and Metabolism, Uppsala University, Uppsala, Sweden; ${ }^{5}$ Department of Clinical Sciences, Intervention and Technology (CLINTEC), Karolinska Institutet, Karolinska University Hospital, Stockholm, Sweden; ${ }^{6}$ Department of Neurobiology, Care Sciences and Society, Division of Clinical Nutrition, Karolinska Institutet, and Clinical Nutrition and Dietetics, Karolinska University Hospital, Stockholm, Sweden
\end{abstract}

\section{Abstract}

Background: Many diseases striking old adults result in eating difficulties. Indications for selecting individuals for percutaneous endoscopic gastrostomy (PEG) are unclear and everybody may not benefit from the procedure.

Objective: The aim of this study was to evaluate indications for and survival after PEG insertion in patients older than 65 years.

Design and Methods: A retrospective analysis including age, gender, diagnosis, indication, and date of death was made in 201 consecutive individuals, 94 male, mean age $79 \pm 7$ years, who received a nutritional gastrostomy.

Results: Dysphagia was present in $86 \%$ of the patients and stroke was the most common diagnosis (49\%). Overall median survival was 123 days and 30-day mortality was $22 \%$. Patients with dementia and $\mathrm{Mb}$ Parkinson had the longest survival (i.e. 244 and 233 days), while those with other neurological diseases, and head and neck malignancy had the shortest (i.e. 75 and 106 days). There was no difference in mortality in patients older or younger than 80 years, except in patients with dementia.

Conclusions: Old age should not be a contraindication for PEG. A high 30-day mortality indicates that there is a need of better criteria for selection and timing of PEG insertion in the elderly.

Keywords: elderly; enteral nutrition; gastrostomy; mortality; PEG; survival

Received: 26 January 201 I; Revised: I3 June 20II; Accepted: 23 June 20I I; Published: 20 July 201 I

$\mathrm{M}$ any older patients have nutritional problems caused by eating difficulties that may be due to somatic diseases, frailty, or fatigue. When oral nutrition no longer is possible or sufficient, enteral nutrition (tube feeding) is an option especially in patients with a functional gastro-intestinal tract. Percutaneous endoscopic gastrostomy (PEG) is the preferential route when the treatment is expected to last for a longer period of time since it is associated with less treatment failures and better nutritional status than nasogastric tube feeding (NGT) (1). Moreover, the risk for complications is less with PEG than with NGT $(1,2)$. PEG was originally described for pediatric use (3), but is today the most common way of supplying artificial enteral nutrition also in adults including the elderly. The intent by using artificial enteral nutrition may be to increase quality of life or to prolong survival. However, many studies report 30-day mortality figures after placement of PEG of around $20 \%(4-8)$; that is, figures that would be unacceptable for any other surgical procedure both from a patient and a health-economical perspective. There is a need to find clinical variables that may facilitate the decision process in order to find the patients that may benefit most from the procedure. In a review, Mitchell and Tetroe (9) pointed out some factors that were related to increased mortality after PEG in patients older than 
65 years, e.g. old age, malignancy, male gender, and hypoalbuminemia. A recent study from Blomberg et al. showed that low albumin, high CRP, age $\geq 65$ years, and body mass index $(\mathrm{BMI})<18.5$ were associated with increased 30-day mortality after PEG insertion. Patients with a combination of low albumin and high CRP levels had a mortality rate of $20 \%$ compared with $<3 \%$ in patients with normal values (10).

The aim of this study was to examine the indications and survival in a consecutive group of elderly individuals who had received PEG.

\section{Methods}

Data for this study was collected between November 2001 and June 2002. Included were all consecutive patients, 65 years or older, that underwent PEG insertion from January 1997 to December 2000 at the Endoscopy Unit at Karolinska University Hospital, Huddinge. A retrospective survey of four different patient chart systems was carried out and the last day for the follow-up was in April 2002. Data on diagnosis, indication for PEG, operating time, removal time, or time of death were collected. The PEG insertion was performed in the endoscopy suite using intravenous conscious sedation and local anesthesia. The pull procedure was used to place a $20 \mathrm{Fr}$ silicon tube with a semirigid inner bumper under endoscopic control. Antibiotic prophylaxis (Cefuroxim) was administered intravenously prior to the procedure. Data of weight and height were not available. Thirteen patients that received their PEG for drainage of an obstruction or for medical supplementation were excluded. For one patient, the time of insertion of the PEG was not noted. In 10 of the remaining 201 patients, the date for removal of the PEG was not retrievable. After these exclusions, the survival analysis was calculated on 191 patients.

\section{Statistics and ethics}

Data are presented as mean $( \pm \mathrm{SD})$ and median with range and 95\% confidence intervals. For survival analysis we used the Log-rank test and Kaplan Meier curves (SPSS and Statistica software programs). The study followed local ethic considerations conformed to the Helsinki declaration.

\section{Results}

There were 201 patients, 65 years or older, that received a PEG for nutritional support between 1997 and 2000 at the Karolinska University Hospital, Huddinge, 107 males, 94 women with a mean age of 79 years (range 65-95). The patients were categorized into seven diagnosis groups: stroke, dementia, Mb Parkinson, other neurological diseases (mainly amyotrophic lateral sclerosis [ALS]), malignancies with or without dysphagia, and miscellaneous. Moreover, they were categorized into three main groups of indications: dysphagia, inability to eat (as a consequence of extreme weakness, motor and mobility problem, and fatigue due to the illness), and nutritional support (EN was used in combination to oral intake). Many patients had multiple diseases. Diagnoses of the patients and the indications for PEG are shown in Table 1. Stroke was the most common diagnosis (49\%) and malignancies with dysphagia were the second most common (16\%). In $86 \%$ of the patients dysphagia was the indication for PEG.

Median survival was 123 days with a range of $0-1713$ days (Fig. 1). Survival in the various diagnosis groups is shown in Table 2. The 30-day mortality in the total patient population was $22 \%$ and the 90 -day mortality was $42 \%$. There were 44 and $33 \%$ of the patients that were still alive after 6 months and after 12 months, respectively. Patients with $\mathrm{Mb}$ Parkinson and dementia had the longest survival while the patients with other neurological diseases and malignant esophageal obstructions had the shortest, with a 1-year mortality of $77 \%$ (Table 2).

When patients were dichotomized according to age over and under 80 years there was no difference in 1-year mortality in the whole group, i.e. 70 and $64 \%$, respectively (ns). Except for patients with dementia, where the corresponding figure for 1-year mortality was 33 and $73 \%$, respectively ( $p=0.025$ ) there was no age-related difference in the various diagnostic subgroups (data not shown).

Twenty patients had their PEG removed after a mean of 300 days and a median of 200 days. Thirteen of these 20 patients were still alive at the time of the last follow-up in the year 2002.

\section{Discussion}

This retrospective survey over older patients that received PEG for nutritional treatment found just stroke to be the most common diagnosis and dysphagia to be responsible for around $90 \%$ of the indications. Median survival was around 4 months.

Most studies on this subject include adults of all ages and the mean age is usually around 65 years. We report data from patients above 65 years. In the literature there seems to be little agreement on whether to report indication or diagnosis for a decision to place a PEG. The prognosis, treatment effect, and survival differed between the diagnoses. There were different indications for PEG insertion within each diagnoses and knowing the indication seems important for planning of medical and nursing resources needed after the hospital stay. In this study both indications and diagnoses are reported. Several of the findings confirm what others have reported regarding underlying disease, survival, and mortality. Neurological disease was the dominating cause for PEG insertion followed by malignancies in the upper GI tract $(5,11)$. In the study by Callahan on 150 elderly patients, $41 \%$ suffered from stroke, $35 \%$ had neuro-degenerative disorders, and 13\% had cancer. 


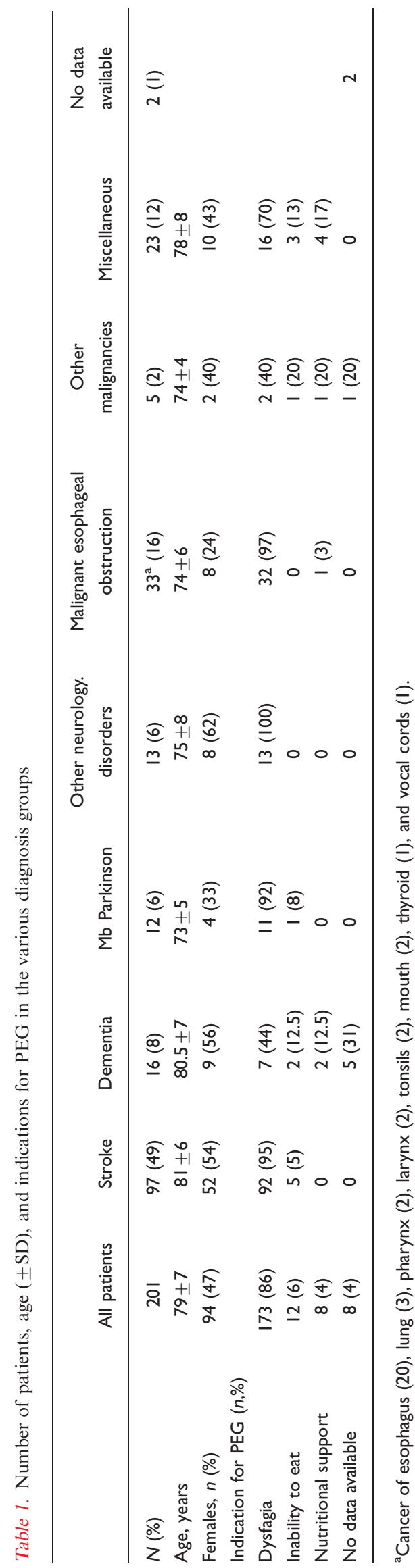

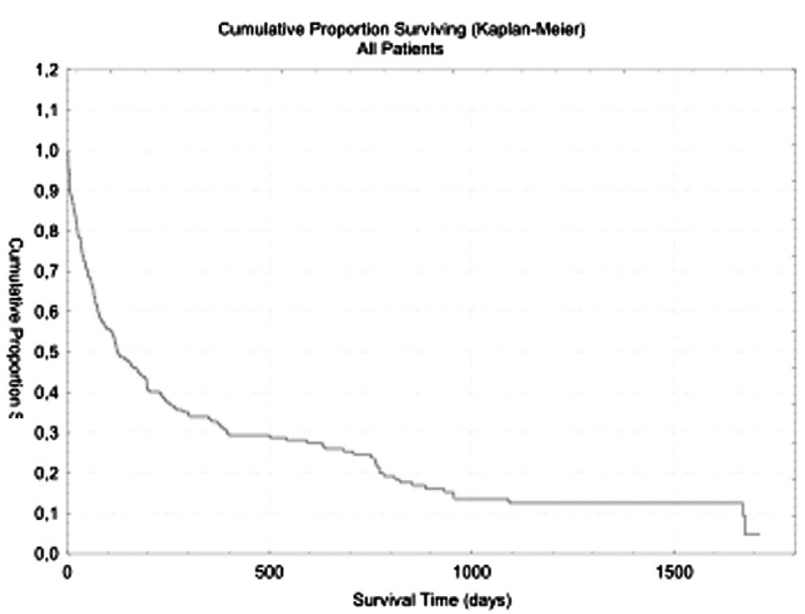

Fig. 1. Survival time, all patients, $n=191$.

When it comes to survival it appears that results from the present study deviates from previous reports. This may reflect different treatment strategies. Not only the decision to start nutritional support by PEG, but also the timing of this decision in relation to the patients clinical status is of great importance for the survival outcome. In our study the median survival time was 123 days, which is shorter than usually presented. For example, in the study by Fisman et al. (6) the median survival was 210 days in 175 patients, all above 65 years of age. The 95 patients with stroke had a median survival of 119 days, which is less than half of the median survival time of 305 days reported by James et al. (12) in 126 patients with stroke and dysphagia and a similar mean age of 80 years. Such differences may reflect a tendency to decide on PEG insertion later in the disease process, rather than variations in the care of the patients after the surgical procedure. The effect of timing and method of enteral feeding for dysphagic stroke patients was studied in the FOOD trial (13). The authors concluded that early tube feeding might reduce case fatality but at the expense of increasing the proportion surviving with poor outcome. Therefore early initiation of PEG feeding was not supported (13). Since only those patients were enrolled in whom the responsible clinician was uncertain of the best feeding practice, the results from this multicentre trial have to be interpreted with caution.

The results from the FOOD trial and other studies on EN in stroke patients with dysphagia are reviewed and analyzed in the ESPEN guidelines on enteral nutrition for geriatric patients. Treatment with EN is recommended as soon as possible unless there are compelling reasons against it in patients with neurological dysphagia. For long-term nutritional support PEG should be preferred to NGT, since it is associated with less treatment failures and better nutritional status (1). Studies on the natural course of dysphagia after stroke show that spontaneous remission of the swallowing difficulty occurs 7-14 days 
Table 2. Median survival in days and 1-,3-, 6-, and 12-month mortality for various diagnostic groups, $n=191$

\begin{tabular}{|c|c|c|c|c|c|}
\hline \multirow{3}{*}{ Median survival in days $(95 \% \mathrm{Cl})$} & & \multicolumn{4}{|c|}{ Mortality (\%) } \\
\hline & & \multicolumn{4}{|c|}{ Days } \\
\hline & & 30 & 90 & 180 & 365 \\
\hline Dementia $(n=16)$ & $244(49-439)$ & 25 & 37 & 37 & 58 \\
\hline Mb Parkinson $(n=\mathrm{II})$ & $233(0-660)$ & 9 & 18 & 36 & 55 \\
\hline Miscellaneous $(n=19)$ & $130(45-215)$ & 17 & 39 & 61 & 61 \\
\hline Stroke $(n=95)$ & $119(15-223)$ & 22 & 46 & 55 & 67 \\
\hline Malignant obstruction $(n=3 \mathrm{I})$ & $106(63-149)$ & 23 & 42 & 68 & 77 \\
\hline Other neurological diseases $(n=13)$ & $75(15-135)$ & 23 & 54 & 62 & 77 \\
\hline Other malignancy $(n=4)$ & - & - & - & - & - \\
\hline No data available $(n=2)$ & - & - & - & - & - \\
\hline All patients $(n=191)$ & $123(74-172)$ & 22 & 42 & 56 & 67 \\
\hline
\end{tabular}

after the acute event in $73 \%-86 \%$ of the patients $(4,14$, 15). Intensive swallowing therapy is also recommended to accompany the treatment (1).

Requests for PEG in order to facilitate care are prevalent; that is, the staff in geriatric and elderly care institutions tend to prefer PEG to a temporary nasogastric tube or to the time-consuming oral feeding. It has been reported that one-third of patients received a PEG due to demands from the community (16). Considerations like this may have implications for the survival length in populations with PEG.

Dementia is a diagnosis where the benefits of placing a PEG are questioned. The decision to place a PEG in a patient with dementia must be taken with ethical considerations $(11,17-21)$. ESPEN guidelines do not recommend enteral nutrition to persons with severe dementia due to more risks than benefits for persons with severe dementia and only occasionally in early and moderate dementia to ensure adequate energy and nutrient supply and to prevent undernutrition (22). A Cochrane review by Sampson et al. confirmed this and concluded that there is insufficient evidence to suggest that enteral nutrition is beneficial in patients with advanced dementia (23). The 244 days of median survival does not support previous observations of a worsened prognosis for patients with dementia and this may reflect a tendency for selecting mainly mildly demented otherwise healthy patients for PEG insertion. The tripled short-term mortality in patients combining dementia and age above 80 years indicate that high age should be a precaution for the decision of PEG even in patients with milder forms of dementia.

A limitation in this survey is that we have no information about the patients' cognitive status at the time when the PEG insertion took place. Further, our data do not allow any conclusions about quality of life for the patients that received PEG. To our knowledge no studies so far have addressed this question.
In accordance with previous reports, a diagnosis of malignancy carried a higher risk of short survival (6, 9, 24). However, the difference was less accentuated in the present study (106 vs. 130 days), when for example compared to a study by Fisman et al. (6) that reported a survival of 137 days in patients with malignant disease versus 321 days in patients with non-malignant disease. It should be acknowledged that PEG insertion due to malignant esophageal obstruction could be justified by palliative reasons even when survival is expected to be short.

Patients with ALS usually present with a long survival after PEG insertion $(9,25)$. In our study the 13 patients with 'other neurological diseases' (12 with ALS) had the shortest survival time of all. However, the current patients were notably older $(75 \pm 8$ years) compared to those presented by Mitchell and Tetroe (9) and Chio et al. (25) (60 and 62 years).

Not unexpectedly, patients older than 80 had a slightly worse prognosis than those younger than 80 , i.e. in line with other reports $(4,26-28)$. This age effect was most pronounced in the patients with dementia. Otherwise the age effect appeared so small that it would not be advisable to identify age as a clear unfavorable factor for PEG in this unselected consecutive group of elderly patients.

The overall 30-day mortality of $22 \%$ is well in accordance with published data $(6,29)$, whereas there are reports on even higher mortality figures of up to $28 \%$ $(11,30)$. The high short-term mortality in this elderly population may not be due to procedure-related complications, but rather to the decisions of PEG placement taken late in the disease course. The 90-day mortality of $42 \%$ and 180 day figure of $56 \%$ also corresponds well to published data of 44 and $52 \%$ (11). In the review by Tetroe and Mitchell (9) the 1-year mortality was $62 \%$, well in line with our figure of $67 \%$ and other reports of 61 
and $66 \%(9,6,30)$. Callahan et al. showed a 1-year mortality of $50 \%(5)$.

We may conclude that there is a major variability in survival after PEG insertion in elderly patients that suffer from various diseases. The high 30-day mortality of $22 \%$ in this study is in accordance with published data, but median survival time appeared to be shorter than previously reported. Dementia was not found to be a negative prognostic factor for survival in this population, especially not in those below the age of 80 years. However, the limited access to patient data, such as disease severity and nutritional state, does not allow a deeper analysis on which clinical variables, which would be discriminative for selecting patients, would benefit most from the procedure. According to the recent study by Blomberg et al., it should not be recommended to make PEG insertion in patients with CRP levels and low albumin indicating ongoing inflammation (10).

There is still an urgent need for developing ways to decide the risk/benefit ratio in the individual patient in order to optimize the timing and route of nutritional support. Especially in this field, ethical consideration is a challenge for the scientific approach, but such efforts would need prospective randomized controlled studies, which most likely are not accepted due to ethical considerations. However, future studies that include questions regarding quality of life are possible to perform and are needed to elucidate the ethical aspects of enteral nutrition treatment in geriatric patients.

\section{Conflict of interest and funding}

The authors have not received any funding or benefits from industry or elsewhere to conduct this study.

\section{References}

1. Volkert D, Berner YN, Berry E, Cederholm T, Coti Bertrand P, Milne A, et al. ESPEN guidelines on enteral nutrition: geriatrics. Clin Nutr 2006; 25: 330-60.

2. Norton B, Homer-Ward M, Donnelly MT, Holmes GKT. A randomised prospective comparison of percutaneous endoscopic gastrostomy and nasogastric tube feeding after acute dysphagic stroke. BMJ 1996; 312: 13-6.

3. Gauderer MWL, Ponsky JL, Izant RJ. Gastrostomy without laparotomy; a percutaneous endoscopic technique. J Pediat Surg 1980; 15: 872-5.

4. Skelly RH, Kupfer RM, Metcalfe ME, Allison SP, Holt M, Hull MA, et al. Percutaneous endoscopic gastrostomy (PEG): change in practice since 1988. Clin Nutr 2002; 21: 389-94.

5. Callahan CM, Haag KM, Weinberger M, Tierney WM, Buchanan NN, Stump TE, et al. Outcomes of percutaneous endoscopic gastrostomy among older adults in a community setting. J Am Geriatr Soc 2000; 48: 1048-54.

6. Fisman DN, Levy AR, Gifford DR, Tamblyn R. Survival after percutaneous endoscopic gastrostomy among older residents of Quebec. J Am Geriatr Soc 1999; 47: 349-53.
7. Abuksis G, Mor M, Segal N, Shemesh I, Plout S, Sulkes J, et al. Percutaneous endoscopic gastrostomy: high mortality rates in hospitalized patients. Am J Gastroenterol 2000; 95: 128-32.

8. Ha L, Hauge T. Percutaneous endoscopic gastrostomy (PEG) for enteral nutrition in patients with stroke. Scand J Gastroenterol 2003; 9: 962-6.

9. Mitchell SL, Tetroe JM. Survival after percutaneous endoscopic gastrostomy placement in older persons. J Gerontol A Biol Sci Med Sci 2000; 55: M735-M739.

10. Blomberg J, Lagergren P, Martin L, Mattsson F, Lagergren J. Albumin and C-reactive protein levels predict short-term mortality after percutaneous endoscopic gastrostomy in a prospective cohort study. Gastrointest Endosc 2010 Nov 11. doi:10.1016/j.gie.2010.09.012

11. Sanders DS, Carter MJ, D'Silva JD, James G, Bolton RP, Bardhan KD. Survival analysis in percutaneous endoscopic gastrostomy feeding: a worse outcome in patients with dementia. Am J Gastroenterol 2000; 95: 1472-5.

12. James A, Kapur K, Hawthorne AB. Long-term outcome of percutaneous endoscopic gastrostomy feeding in patients with dysphagic stroke. Age Ageing 1998; 27: 671-6.

13. FOOD Trial Collaboration. Effect of timing and method of enteral tube feeding for dysphagic stroke patients (FOOD): a multicentre randomised controlled trial. Lancet 2005; 365: 764-72.

14. Smithard DG, O'Neill PA, England RE, Park CL, Wyatt R, Martin DF, et al. The natural history of dysphagia following a stroke. Dysphagia 1997; 12: 188-93.

15. Gordon C, Hewer RL, Wade DT. Dysphagia in acute stroke. Dysphagia 1985; 295: 411-4.

16. Van Rosendaal GMA, Verhoef MJ, Kinsella TD. How are decisions made about the use of percutaneous endoscopic gastrostomy for long-term nutritional support? Am J Gastroenterol 1999; 94: 3225-8.

17. Nair S, Hertan H, Pitchumoni CS. Hypoalbuminemia is a poor predictor of survival after percutaneous endoscopic gastrostomy in elderly patients with dementia. Am J Gastroenterol 2000; 95: $133-6$.

18. Mitchell SL, Kiely DK, Lipsitz LA. The risk factors and impact of survival of feeding tube placement in nursing home residents with severe cognitive impairment. Arch Intern Med 1997; 157: $327-32$.

19. Murphy LM, Lipman TO. Percutaneous endoscopic gastrostomy does not prolong survival in patients with dementia. Arch Intern Med 2003; 163: 1351-3.

20. Dharmarajan TS, Unnikrishnan D, Pitchumoni CS. Percutaneous endoscopic gastrostomy and outcome in dementia. Am J Gastroenterol 2001; 96: 2556-63.

21. Sampson EL, Candy B, Jones L. Enteral tube feeding for older people with advanced dementia. Cochrane Database Syst Rev. 2009; Apr 15: CD007209.

22. Körner U, Bondolfi A, Bühler E, MacFie J, Meguid MM, Messing B, et al. Ethical and legal aspects of enteral nutrition. ESPEN guidelines. Clin Nutr 2006; 25: 196-202.

23. Gillick MR. Rethinking the role of tube feeding in patients with advanced dementia. NEJM 2000; 342: 206-10.

24. Cortez-Pinto H, Correia AP, Camilo ME, Tavares L, De Moura MC. Long-term management of percutaneous endoscopic gastrostomy by a nutritional support team. Clin Nutr 2002; 2 : $27-31$.

25. Chiò A, Finocchiaro E, Meineri P, Bottacchi E, Schiffer D. Safety and factors related to survival after percutaneous endoscopic gastrostomy in ALS. ALS Percutaneous Endoscopic Gastrostomy Study Group. Neurology 1999; 53: 1123-5. 
26. Paillaud E, Bories PN, Merlier I, Richardet JP, Jeanfaivre V, Campillo B. Facteurs pronostiques de la survie à court et long terme après pose d'une gastrostomie percutanée endoscopique chez des malades âgés hospitalisés (full text in English on http:// www.e2med.com/gcb). Gastroenterol Clin Biol 2002; 26: 443-7.

27. Kaw M, Sekas G. Long-term follow-up of consequences of percutaneous endoscopic gastrostomy (PEG) tubes in nursing home patients. Dig Dis Sci 1994; 39: 738-43.

28. Weaver JP, Odell P, Nelson C. Evaluation of the benefits of gastric tube feeding in an elderly population. Arch Fam Med 1993; 2: 953-6.

29. Raha SK, Woodhouse K. The use of percutaneous endoscopic gastrostomy (PEG) in 161 consecutive elderly patients. Age Ageing 1994; 23: 162-3.
30. Löser C, Wolters S, Fölsch UR. Enteral long-term nutrition via percutaneous endoscopic gastrostomy (PEG) in 210 patients. Dig Dis Sci 1998; 43: 2549-57.

\section{*Gerd Faxen-Irving}

Karolinska University Hospital

Huddinge

SE-14| 86 Stockholm, Sweden

Tel: + (46) 8 5858607|

$\mathrm{Fax}+(46) 858580540$

Email: gerd.faxen.irving@ki.se 